\title{
Magnetically confined bound states in Rashba systems
}

\author{
Flavio Ronetti, Kirill Plekhanov, Daniel Loss ${ }^{\circ}$, and Jelena Klinovaja \\ Department of Physics, University of Basel, Klingelbergstrasse 82, CH-4056 Basel, Switzerland
}

(Received 14 November 2019; revised manuscript received 6 April 2020; accepted 15 May 2020; published 8 June 2020)

\begin{abstract}
A Rashba nanowire is subjected to a magnetic field that assumes opposite signs in two sections of the nanowire, and, thus, creates a magnetic domain wall. The direction of magnetic field is chosen to be perpendicular to the Rashba spin-orbit vector such that there is only a partial gap in the spectrum. Nevertheless, we prove analytically and numerically that such a domain wall hosts a bound state whose energy is at bottom of the spectrum below the energy of all bulk states. Thus, this magnetically confined bound state is well-isolated and can be accessed experimentally. We further show that the same type of magnetic confinement can be implemented in two-dimensional systems with strong spin-orbit interaction. A quantum channel along the magnetic domain wall emerges with a nondegenerate dispersive band that lies energetically below the bulk states. We show that this magnetic confinement is robust against disorder and various parameter variations.
\end{abstract}

DOI: 10.1103/PhysRevResearch.2.022052

\section{INTRODUCTION}

The possibility of confining electrons to manipulate their quantum state plays an extremely important role in condensed matter physics and paves the way for various quantum computing schemes [1-4]. The prime example are quantum dots where the confinement can be generated by external gates or intrinsically via mismatch of band gaps. The confinement can also result from nonuniform superconducting gaps giving rise to Andreev bound states [5-9]. Other ways to confine states are based on interfaces or domain walls that separate regions of different phases, well-known examples being Jackiw-Rebbi fermions [10-18] and, in particular, Majorana bound states in proximitized nanowires (NWs) with Rashba spin-orbit interaction (SOI) [19-33].

It is then natural to ask if there are further ways to confine electrons and thereby open up new platforms for bound states. Motivated by this question, we consider systems with uniform Rashba SOI in the presence of a nonuniform magnetic field with a domain wall. If the direction of the magnetic field is perpendicular to the Rashba SOI vector [34-37], we find a bound state that lies in energy below all extended states. Remarkably, such a magnetically confined bound state occurs even in the regime where the Zeeman energy is much smaller than the SOI energy. While for analytical calculations a sharp transition of magnetic field is considered, numerically we confirm that the bound state and bulk states are energetically well-separated even for smooth magnetic domain walls. We also show that the bound states are robust against disorder and various parameter variations. Finally, we consider a

Published by the American Physical Society under the terms of the Creative Commons Attribution 4.0 International license. Further distribution of this work must maintain attribution to the author(s) and the published article's title, journal citation, and DOI. two-dimensional (2D) Rashba layer and show that, similarly to the NW, a one-dimensional quantum channel, whose dispersion lies energetically below any other bulk states, arises at the interface between two regions of opposite perpendicular magnetic fields. The breaking of the inversion symmetry in the spectrum opens access to the ratio between Rashba and Dresselhaus SOI terms. The setups proposed here can be experimentally implemented by placing a Rashba system on ferromagnets with magnetic domains.

\section{MODEL}

We consider a Rashba NW aligned along the $x$ axis and subjected to a nonuniform magnetic field, see Fig. 1. The kinetic part of the Hamiltonian is given by (with $\hbar=1$ )

$$
H_{0}=\int d x \Psi_{\sigma}^{\dagger}(x)\left[\frac{-\partial_{x}^{2}}{2 m}-\mu-i \alpha_{R} \partial_{x} \sigma_{y}\right]_{\sigma \sigma^{\prime}} \Psi_{\sigma^{\prime}}(x),
$$

where $\Psi_{\sigma}(x)$ is the annihilation operator acting on an electron with spin $\sigma / 2= \pm 1 / 2$ at position $x$ of the $\mathrm{NW}$ and $\sigma_{i}$ is the Pauli matrix acting on the electron spin. Here, $\mu$ is the chemical potential and $m$ is the effective mass. The Rashba SOI, assumed to be uniform, is characterized by the SOI vector $\boldsymbol{\alpha}_{R}=\left(0, \alpha_{R}, 0\right)$, which is aligned along the $y$ direction. In addition, we also define the SOI momentum (energy) $k_{\mathrm{so}}=$ $m \alpha_{R}\left(E_{\mathrm{so}}=k_{\mathrm{so}}^{2} / 2 m\right)$.

To generate a domain wall, we apply an external magnetic field $\mathbf{B}$ perpendicular to the SOI vector $\boldsymbol{\alpha}_{R}$, i.e., along the $z$ axis. We assume that $\mathbf{B}$ has opposite directions in the two regions $x>0$ and $x<0$, thus, allowing for the existence of localized bound states at the domain wall at $x=0$. In order to address this nonuniform magnetic field, we introduce a position-dependent Zeeman term given by

$$
H_{Z}=\int d x \Delta_{Z}(x) \Psi_{\sigma}^{\dagger}(x)\left(\sigma_{z}\right)_{\sigma \sigma^{\prime}} \Psi_{\sigma^{\prime}}(x) .
$$


(a)
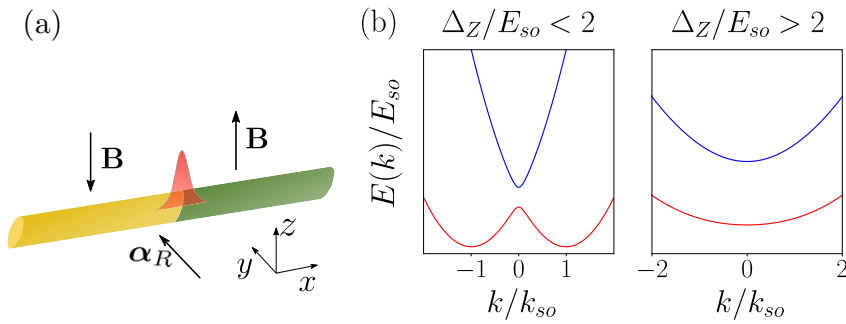

FIG. 1. (a) The NW aligned in $x$ direction with uniform Rashba SOI vector $\boldsymbol{\alpha}_{R}$ pointing along the $y$ axis. A magnetic field $\mathbf{B}$ is applied perpendicular to $\boldsymbol{\alpha}_{R}$, i.e. along the $z$ axis, resulting in a partial gap in the bulk spectrum. To generate a magnetic domain wall hosting a bound state (red), the directions of $\mathbf{B}$ are chosen to be opposite for $x>0$ (green region) and for $x<0$ (yellow region). (b) Bulk spectrum for uniform magnetic field for two different regimes of relative strength between Zeeman energy $\Delta_{Z}$ and Rashba SOI energy $E_{\text {so. }}$. A gap at zero momentum $k=0$ given by $\Delta_{Z}$ separates the two bands. If $\Delta_{Z} / E_{\mathrm{so}}<2$, the lowest band has a local maximum at $k=0$ and a local minimum around $k \sim k_{\mathrm{so}}$. If $\Delta_{Z} / E_{\mathrm{so}}>2$, only a single global minimum exists at $k=0$.

To provide an analytical treatment of this model, we focus on a specific functional dependence of the Zeeman energy, $\Delta_{Z}(x)=\Delta_{Z} \operatorname{sgn}(x)$ with $\Delta_{Z}=g \mu_{B} B$, where $g$ is the $g$ factor and $\mu_{B}$ is the Bohr magneton. This particular choice mimics an abrupt change of direction at the interface $x=0$. The effects of a smooth transition can be treated by numerical simulations, where the smooth change of the magnetic field can be described, e.g., as $\Delta_{Z}(x)=\Delta_{Z} \tanh (x / \delta)$. Here, the parameter $\delta$ characterizes the width of the domain wall. The abrupt change at $x=0$ corresponds to the limit $k_{\mathrm{so}} \delta \rightarrow 0$.

The total Hamiltonian is given by $H=H_{0}+H_{Z}$. For a uniform magnetic field $\Delta_{Z}(x) \equiv \Delta_{Z}$, the energy spectrum consists of two bands separated by a gap,

$$
E_{ \pm}(k)=\left(k^{2}+k_{\mathrm{so}}^{2} \pm 2 \sqrt{k^{2} k_{\mathrm{so}}^{2}+m^{2} \Delta_{Z}^{2}}\right) / 2 m
$$

where we assumed $\mu=-E_{\mathrm{so}}$. We note that the shape of the bands changes significantly depending on whether the dominant energy scale is $E_{\text {so }}$ or $\Delta_{Z}$. In the first case, $E_{-}(k)$ has a local maximum at $k=0$ as well as a two minima close to $k \sim k_{\text {so }}$. In the opposite case, only a single global minimum exists at $k=0$ [see Fig. 1(b)]. The transition between these two regimes occurs at $\Delta_{Z} / E_{\mathrm{so}}=2$. In general, the bottom of the lowest band $E_{-}(k)$, denoted as $E_{1}$, moves according to the following expression:

$$
E_{1}=\left\{\begin{array}{ll}
-\Delta_{Z}^{2} / 4 E_{\mathrm{so}}, & \Delta_{Z}<2 E_{\mathrm{so}} \\
E_{\mathrm{so}}-\Delta_{Z}, & \Delta_{Z} \geqslant 2 E_{\mathrm{so}}
\end{array} .\right.
$$

This value corresponds to the minimal energy of bulk electrons in the NW. Surprisingly, as we will show in the following, a bound state localized at the domain wall at $x=0$ can exist even at energies below $E_{1}$.

\section{BOUND STATE AT THE DOMAIN WALL}

In order to demonstrate the existence of a bound state at the interface $x=0$ for a sharp domain wall analytically, one has to solve the Schrodinger equation $\mathcal{H}(x) \psi(x)=E \psi(x)$, where we focus on solutions below the bottom of the band $E<E_{1}$, see Fig. 2. Here, $\mathcal{H}(x)$ is the Hamiltonian density associated with $H$ and $\psi(x)=\left(\psi_{\uparrow}(x), \psi_{\downarrow}(x)\right)^{T}$ is a $2 \mathrm{D}$ spinor. The eigenfunction of $\mathcal{H}$ in each of the two separate regions has the following form: $\psi(x)=\left(v_{\uparrow}(k), v_{\downarrow}(k)\right)^{T} e^{i k x}$, where $k$ is a complex number obtained by solving the equation $E_{ \pm}(k)=E$ in the regime $E<E_{1}$, see Eq. (3). Indeed, an exponential decay required for having a bound state is encoded in the imaginary part of $k$ : the latter should be positive (negative) for $x>0(x<0)$, in order to find a normalizable solution localized at $x=0$. The solution is constructed by matching the corresponding wave functions at the interface $x=0$. We find that there exists a nondegenerate bound state with energy $E_{\mathrm{BS}}<E_{1}$ under the condition $\Delta_{Z} / E_{\mathrm{so}}<4$. The expression for the energy $E_{\mathrm{BS}}$ of this bound state is involved but still can be
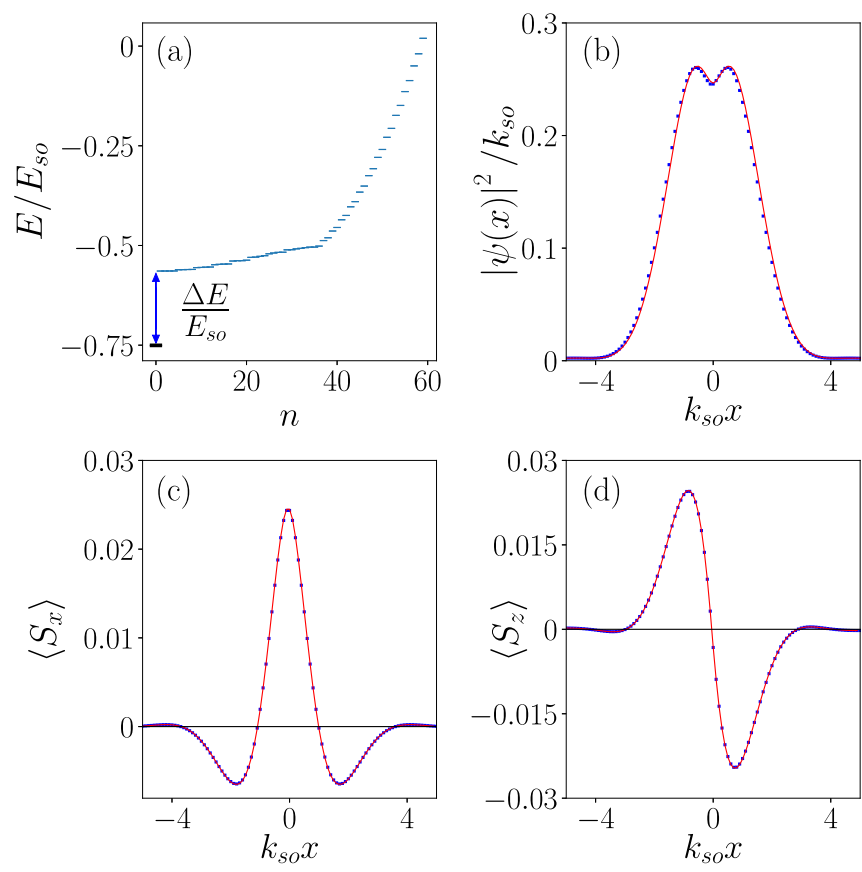

FIG. 2. (a) The lowest energy states of a Rashba NW with a Zeeman field $\left(\Delta_{Z} / E_{\mathrm{so}}=1.5\right)$ that changes sign at $x=0$, obtained by numerical diagonalization of a system with 1300 sites. The lowest energy state (black line), which corresponds to the nondegenerate bound state at $x=0$, is energetically well separated by $\Delta E$ from the bulk spectrum. (b) The probability density $|\psi(x)|^{2}$ of the bound state, (c) the $x$ component, and (d) the $z$ component of its spin polarization. The analytical (red lines) and numerical results (blue dots) are in excellent agreement. 
found analytically:

$$
\frac{E_{\mathrm{BS}}}{E_{\mathrm{so}}}=-\frac{2^{\frac{2}{3}}\left[3\left(\frac{\Delta_{Z}}{E_{\mathrm{so}}}\right)^{2}+2\right]+\frac{2^{\frac{1}{3}}}{12}\left[27\left(\frac{\Delta_{Z}}{E_{\mathrm{so}}}\right)^{4}+72\left(\frac{\Delta_{Z}}{E_{\mathrm{so}}}\right)^{2}+3 \sqrt{81\left(\frac{\Delta_{Z}}{E_{\mathrm{so}}}\right)^{8}+48\left(\frac{\Delta_{Z}}{E_{\mathrm{so}}}\right)^{6}}+32\right]^{\frac{2}{3}}}{3 \sqrt[3]{27\left(\frac{\Delta_{Z}}{E_{\mathrm{so}}}\right)^{4}+72\left(\frac{\Delta_{Z}}{E_{\mathrm{so}}}\right)^{2}+3 \sqrt{81\left(\frac{\Delta_{Z}}{E_{\mathrm{so}}}\right)^{8}+48\left(\frac{\Delta_{Z}}{E_{\mathrm{so}}}\right)^{6}}+32}}+\frac{2}{3} .
$$

(a)
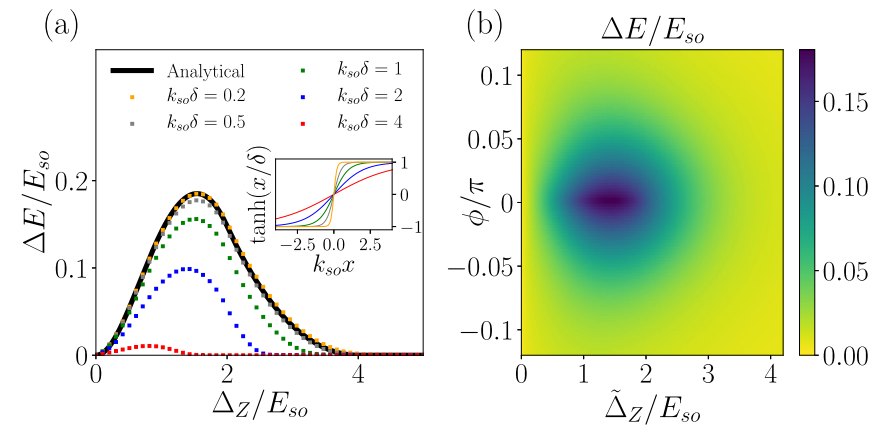

FIG. 3. (a) Energy separation $\Delta E / E_{\mathrm{so}}$ between the bound state and the lowest bulk state as a function of $\Delta_{Z} / E_{\mathrm{so}}$ for different sizes of the domain wall $\delta$. The different colors are assigned to points obtained by numerical simulation. Black solid line corresponds to the analytical result obtained in the sharp domain wall limit. (Inset) The magnetic field profile close to the interface for different value of $\delta$. The larger $\delta$, the smaller is $\Delta E$. Also, the smoother the profile, the smaller is $\Delta_{Z}$ at which the bound state merges with the continuum. (b) Energy separation $\Delta E / E_{\mathrm{so}}$ as a function of the magnitude of magnetic field $\tilde{\Delta}_{Z}$ and the angle $\phi$ that the magnetic field forms with the $z$ axis in the presence of an additional parallel component. This shows the robustness of $\Delta E$ towards tilting of the magnetic field.

If $\Delta_{Z} / E_{\text {so }} \geqslant 4$, the bound state disappears by merging with the bulk spectrum.

Next, we define the energy separation $\Delta E=E_{1}-E_{\mathrm{BS}} \geqslant$ 0 between this bound state and the lowest bulk state [see Fig. 2(a)]. If there is no bound state in the spectrum, we set $\Delta E=0$. In the limit of weak Zeeman field, the bound states splits from the bulk modes quadratically in the Zeeman energy as

$$
\Delta E=\frac{1}{4} \frac{\Delta_{Z}^{2}}{E_{\mathrm{so}}} .
$$

Comparing the analytical solution and the numerical solution obtained in the discretized model, we find excellent agreement (see Fig. 3). The found bound state localized at $x=0$ is the lowest energy state and is well-separated from then extended bulk modes. We also confirm that, as expected, the bound state merges with the bulk states at $\Delta_{Z} / E_{\mathrm{so}}=4$. Even though we focused on the lowest two bands of the NW, identical bound states also appear right below the bottom of higher band pairs. However, the visibility of such bound states is masked by the presence of extended bulk modes from lower bands [38,39].

We note that the configuration described above can be mapped to an equivalent system without Rashba SOI by applying the spin-dependent gauge transformation $\Psi_{\sigma}(x) \rightarrow$ $e^{i \sigma k_{\mathrm{so}} x} \Psi_{\sigma}(x)$ [40]. This transformation eliminates the term $H_{R}$ in the Hamiltonian and changes the Zeeman energy to $\Delta_{Z}(x) \rightarrow \Delta_{Z}(x)\left[\cos \left(2 k_{\mathrm{so}} x\right) \hat{z}+\sin \left(2 k_{\mathrm{so}} x\right) \hat{x}\right]$. The latter corresponds to a helical magnetic field, which could be created either extrinsically by arrays of nanomagnets [40-47] or intrinsically via ordering of nuclear spins or magnetic adatoms due to Ruderman-Kittel-Kasuya-Yosida interaction [48-50]. By analogy, the domain walls occurring in such structures will also host bound states, see the Supplemental Material (SM) [51]. We note that, in this model, a simple physical picture for the bound states can be given. While in the regime of weak magnetic fields a partial gap at $k=0$ opens at high densities, the bottom of the band $E_{1}$ stays almost unaffected. This can be easily understood since the effects of a rapidly rotating field with a period much shorter than the wavelengths of lowdensity modes averages out to zero. However, at the domain wall, where the sign of the magnetic field flips, this averaging does not occur, giving rise to a non-zero local Zeeman term, shifting the bottom of the band down and thereby creating a local potential minimum that traps a bound state [52].

\section{BOUND STATE WAVE FUNCTION AND POLARIZATION}

Numerically, we study the spectrum for arbitrary profiles of magnetic fields and get access to the bound state wave function. In the case of a sharp domain wall, the analytical expression for the wave function of the bound state can be derived. The corresponding probability density is plotted in Fig. 2(b) and compared with the numerical solution. The agreement between the two quantities is excellent. From the analytics, we also obtain the localization length of the bound state

$$
\xi_{\mathrm{BS}}^{-1}=k_{\mathrm{so}} \mathfrak{I m} \sqrt{1+\frac{E_{\mathrm{BS}}}{E_{\mathrm{So}}}+\sqrt{\frac{4 E_{\mathrm{BS}}}{E_{\mathrm{So}}}+\left(\frac{\Delta_{Z}}{E_{\mathrm{So}}}\right)^{2}}},
$$

which, for the parameters of Fig. 2(b), is equal to $\xi_{\mathrm{BS}} \sim$ $1.75 / k_{\mathrm{so}}$. Thus, we have established the existence of a bound state localized within $k_{\text {so }}^{-1}$ around $x=0$ and with an energy separation $\Delta E$ well below the energy of the bulk states.

It is also interesting to study the spin polarization of this bound state, $\left\langle S_{i}(x)\right\rangle=\sum_{\sigma, \sigma^{\prime}} \psi_{\sigma}^{*}(x)\left(\sigma_{i}\right)_{\sigma \sigma^{\prime}} \psi_{\sigma^{\prime}}(x)$ with $i=$ $x, y, z$. We observe that the polarization along the SOI vector $\boldsymbol{\alpha}_{R}$ vanishes, $\left\langle S_{y}(x)\right\rangle=0$, i.e., due to the mirror symmetry [53], the polarization stays orthogonal to $\boldsymbol{\alpha}_{R}$. The other two components are nonzero, see Figs. 2(c) and 2(d). The $x$ component $\left\langle S_{x}(x)\right\rangle$ is symmetric with respect to $x=0$ with a global maximum close to the interface. Away from the interface, $\left\langle S_{x}(x)\right\rangle$ changes sign and reaches its global minimum before vanishing after a length of a few $k_{\text {so }}^{-1}$. The component along the magnetic field, $\left\langle S_{z}(x)\right\rangle$, is odd in $x$ and follows the sign of the magnetic field. 


\section{STABILITY OF THE BOUND STATE}

Numerically, we can study the stability of the bound states away from the sharp domain wall limit. First, we consider a domain wall with smooth transition, modeled by $\Delta_{Z}(x)=$ $\Delta_{Z} \tanh (x / \delta)$, see Fig. 3. The agreement between analytical and numerical results improves as $\delta$ decreases and for $k_{\mathrm{so}} \delta<$ 0.2 the match is almost exact. In the case of smoother transitions with $k_{\mathrm{so}} \delta>0.2$, the bound state merges with the continuum at smaller Zeeman energies (smaller than $\Delta_{Z} / E_{\text {so }}=4$ ). The analytical expression for the energy separation exhibits a maximum $\Delta E / E_{\mathrm{so}} \sim 0.18$ around $\Delta_{Z} / E_{\mathrm{so}} \sim 1.5$. This value is reduced as $\delta$ grows. This opens the door to access the spinorbit energy experimentally: measuring the value of $\Delta_{Z}$ at which the bound state disappears provides an estimate on $E_{\mathrm{so}}$.

Generally, the optimal domain wall width has to be of the order of $\delta \sim k_{\text {so }}^{-1}$, which, for an InSb nanowire with the SOI energy in the range $E_{\mathrm{so}} \sim 0.1-0.5 \mathrm{meV}[33,54]$, implies an upper bound around $\delta \simeq 200 \mathrm{~nm}$. Given $g \sim 20$ [55], a perpendicular magnetic field in the range $B \sim 0.5-2.5 \mathrm{~T}$ guarantees an energy separation between the bound state and bulk states of the order $\Delta E \sim 0.075 \mathrm{meV}(\sim 750 \mathrm{mK})$, which is experimentally accessible with standard low-temperature measurements.

To confirm the robustness of the bound state, we add a small uniform component $B_{\|}$parallel to the SOI vector. As a result, the total field has an angle $\phi=\arctan \left(B_{\|} / B\right)$ with the $z$ axis, see Fig. 3(b). The corresponding Zeeman energy becomes $\tilde{\Delta}_{Z}=g \mu_{B} \sqrt{B^{2}+B_{\|}^{2}}$, where, for simplicity, we assumed an isotropic $g$ factor. As expected, the energy separation between bulk states and the bound state has a maximum in the regime where the domain wall is most pronounced, $\phi=0$. However, there exists a wider range of magnetic field orientations, which we estimate as $|\phi|<\pi / 10$, for which the bound states still exist. In addition, we confirmed numerically the stability against disorder by allowing for fluctuations in the chemical potential and magnetic field (see SM [51]). All this demonstrates that the emergence of the bound states does note rely on fine-tuning of parameters but is a rather stable effect.

\section{QUANTUM CHANNEL ALONG DOMAIN WALL IN RASHBA LAYER}

We extend now our consideration to 2D systems with strong SOI in the presence of a perpendicular magnetic Zeeman field whose sign is opposite in the two regions of the plane separated by the line $x=0$, defining the domain wall, see Fig. 4(a). We assume periodic boundary conditions in the $y$ direction and, thus, the associated momentum $k_{y}$ is a good quantum number. In two dimensions, a bound state localized along the domain wall evolves into an extended 1D channel with the dispersive energy spectrum, see Fig. 4(b).

We consider two configurations for the 2D SOI described by $\mathcal{H}_{R}=-i\left(\alpha_{x} \sigma_{y} \partial_{y}-\alpha_{y} \sigma_{x} \partial_{x}\right)$. In the first one, corresponding to the left panel of Fig. 4(b), the Rashba and Dresselhaus SOI are equal resulting in $\alpha_{x}=0$ [56-64], such that the system is symmetric with respect to $y \rightarrow-y$. The lower energy states, defining the $1 \mathrm{D}$ quantum channel, have a finite separation from the $2 \mathrm{D}$ bulk states (like the bound state in the NW). In this case, the energy dispersion of the channel states acquires
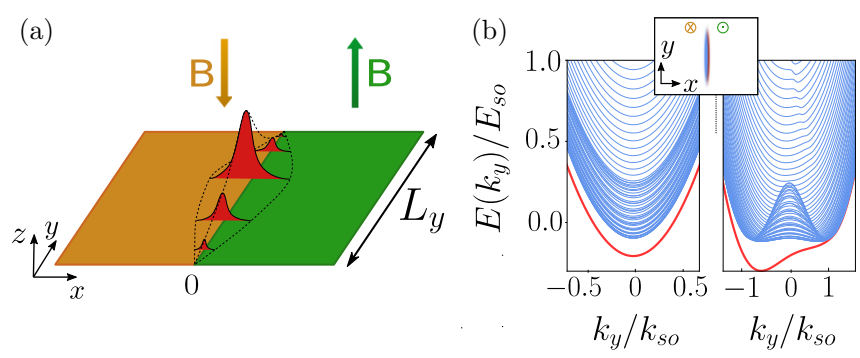

FIG. 4. (a) 2D Rashba layer placed in the $x y$ plane and $\mathbf{B}$ is applied perpendicular to the plane with opposite directions for $x>$ 0 (green region) and for $x<0$ (yellow region) creating a onedimensional domain wall that hosts a quantum channel running along the $y$ axis. (b) Energy spectrum as function of $k_{y}$ in the absence (presence) of Rashba SOI along the $y$ axis is shown on left (right) panel. The red curves describe the spectrum of the nondegenerate quantum channel localized along the domain wall. (Inset) Probability density of the quantum channel states found numerically for a finitesize system.

a simple parabolic form, $E\left(k_{y}\right)=E_{\mathrm{BS}}+k_{y}^{2} / 2 m$, which is symmetric with respect to $k_{y}=0$, with $E_{\mathrm{BS}}$ given in Eq. (5). If $\alpha_{y}$ is finite, the inversion symmetry is broken and the quantum channel dispersion relation is now asymmetric, see the right panel of Fig. 4(b). The largest energy separation occurs at a finite value of momentum and acquires a larger value compared to the previous case. Interestingly, this asymmetry in the energy dispersion provides a test for the presence of a second component of SOI and a way to access its magnitude. Finally, the probability density in real space has similar shape for both configurations [see Fig. 4(b)]: as expected, the quantum channel is extended along the domain wall at $x=0$. This channel is still localized along the domain wall even for curved or closed wall shapes, as long as there is no large in-plane magnetic field parallel to one of the SOI components (see SM [51]).

\section{CONCLUSIONS}

We considered a Rashba NW in the presence of a domain wall created by a perpendicular magnetic Zeeman field, with opposite sign in the two corresponding domains. At the domain wall, a bound state exists whose energy is separated from the lowest bulk modes. This separation persists for smooth domain walls and for a slightly tilted magnetic field. This effect is straightforwardly extended to 2D Rashba layers where the domain wall hosts a quantum channel: a propagating nondegenerate mode with parabolic dispersion. Our predictions can be tested by transport [7-9] and cavity measurements [65]. Finally, moving the magnetic domain walls adiabatically will allow us to move the bound states attached to them.

\section{ACKNOWLEDGMENTS}

This work was supported by the Swiss National Science Foundation, NCCR QSIT, and the Georg H. Endress foundation. This project received funding from the European Union's Horizon 2020 research and innovation program (ERC Starting Grant, Grant No. 757725). 
[1] D. Loss and D. P. DiVincenzo, Phys. Rev. A 57, 120 (1998).

[2] A. Y. Kitaev, Phys.-Usp. 44, 131 (2001).

[3] A. G. Fowler, A. M. Stephens, and P. Groszkowski, Phys. Rev. A 80, 052312 (2009).

[4] C. Kloeffel and D. Loss, Ann. Rev. Condens. Matter Phys. 4, 51 (2013).

[5] J. A. Sauls, Philos. Trans. Roy. Soc. London Series A 376, 20180140 (2018).

[6] E. J. H. Lee, X. Jiang, R. Aguado, G. Katsaros, C. M. Lieber, and S. De Franceschi, Phys. Rev. Lett. 109, 186802 (2012).

[7] K. Grove-Rasmussen, G. Steffensen, A. Jellinggaard, M. H. Madsen, R. Žitko, J. Paaske, and J. Nygârd, Nat. Commun. 9, 2376 (2018).

[8] C. Jünger, A. Baumgartner, R. Delagrange, D. Chevallier, S. Lehmann, M. Nilsson, K. A. Dick, C. Thelander, and C. Schönenberger, Commun. Phys. 2, 76 (2019).

[9] M. Hays, G. de Lange, K. Serniak, D. J. van Woerkom, D. Bouman, P. Krogstrup, J. Nygård, A. Geresdi, and M. H. Devoret, Phys. Rev. Lett. 121, 047001 (2018).

[10] R. Jackiw and C. Rebbi, Phys. Rev. D 13, 3398 (1976).

[11] W. P. Su, J. R. Schrieffer, and A. J. Heeger, Phys. Rev. Lett. 42, 1698 (1979).

[12] R. Rajaraman and J. Bell, Phys. Lett. B 116, 151 (1982).

[13] S. Kivelson and J. R. Schrieffer, Phys. Rev. B 25, 6447 (1982).

[14] A. J. Heeger, S. Kivelson, J. R. Schrieffer, and W. P. Su, Rev. Mod. Phys. 60, 781 (1988).

[15] J. Klinovaja and D. Loss, Phys. Rev. Lett. 110, 126402 (2013).

[16] X. Deng and L. Santos, Phys. Rev. A 89, 033632 (2014).

[17] D. Rainis, A. Saha, J. Klinovaja, L. Trifunovic, and D. Loss, Phys. Rev. Lett. 112, 196803 (2014).

[18] Y. Nishida, L. Santos, and C. Chamon, Phys. Rev. B 82, 144513 (2010).

[19] R. Pawlak, S. Hoffman, J. Klinovaja, D. Loss, and E. Meyer, Prog. Part. Nucl. Phys. 107, 1 (2019).

[20] E. Prada, P. San-Jose, M. W. A. de Moor, A. Geresdi, E. J. H. Lee, J. Klinovaja, D. Loss, J. Nygård, R. Aguado, and L. P. Kouwenhoven, arXiv:1911.04512 (2019).

[21] Y. Oreg, G. Refael, and F. von Oppen, Phys. Rev. Lett. 105, 177002 (2010).

[22] R. M. Lutchyn, J. D. Sau, and S. Das Sarma, Phys. Rev. Lett. 105, 077001 (2010).

[23] A. C. Potter and P. A. Lee, Phys. Rev. B 83, 094525 (2011).

[24] D. Sticlet, C. Bena, and P. Simon, Phys. Rev. Lett. 108, 096802 (2012).

[25] B. I. Halperin, Y. Oreg, A. Stern, G. Refael, J. Alicea, and F. von Oppen, Phys. Rev. B 85, 144501 (2012).

[26] P. San-Jose, E. Prada, and R. Aguado, Phys. Rev. Lett. 108, 257001 (2012).

[27] D. Rainis, L. Trifunovic, J. Klinovaja, and D. Loss, Phys. Rev. B 87, 024515 (2013).

[28] A. Das, Y. Ronen, Y. Most, Y. Oreg, M. Heiblum, and H. Shtrikman, Nat. Phys. 8, 887 (2012).

[29] M. T. Deng, C. L. Yu, G. Y. Huang, M. Larsson, P. Caroff, and H. Q. Xu, Nano Lett. 12, 6414 (2012).

[30] R. M. Lutchyn, E. P. A. M. Bakkers, L. P. Kouwenhoven, P. Krogstrup, C. M. Marcus, and Y. Oreg, Nat. Rev. Mater. 3, 52 (2018).

[31] M. T. Deng, S. Vaitiekenas, E. B. Hansen, J. Danon, M. Leijnse, K. Flensberg, J. Nygård, P. Krogstrup, and C. M. Marcus, Science 354, 1557 (2016).
[32] M.-T. Deng, S. Vaitiekenas, E. Prada, P. San-Jose, J. Nygård, P. Krogstrup, R. Aguado, and C. M. Marcus, Phys. Rev. B 98 085125 (2018).

[33] V. Mourik, K. Zuo, S. M. Frolov, S. R. Plissard, E. P. A. M Bakkers, and L. P. Kouwenhoven, Science 336, 1003 (2012).

[34] P. Středa and P. Šeba, Phys. Rev. Lett. 90, 256601 (2003).

[35] Y. V. Pershin, J. A. Nesteroff, and V. Privman, Phys. Rev. B 69, 121306(R) (2004).

[36] T. Meng and D. Loss, Phys. Rev. B 88, 035437 (2013).

[37] J. Kammhuber, M. C. Cassidy, F. Pei, M. P. Nowak, A. Vuik, O. Gül, D. Car, S. R. Plissard, E. P. A. M. Bakkers, M. Wimmer, and L. P. Kouwenhoven, Nat. Commun. 8, 478 (2017); J. Klinovaja and D. Loss, Eur. Phys. J. B 88, 62 (2015).

[38] C. W. Hsu, B. Zhen, A. D. Stone, J. D. Joannopoulos, and M. Soljačić, Nat. Rev. Mater. 1, 16048 (2016).

[39] D. M. Kennes, N. Müller, M. Pletyukhov, C. Weber, C. Bruder, F. Hassler, J. Klinovaja, D. Loss, and H. Schoeller, Phys. Rev. B 100, 041103(R) (2019).

[40] B. Braunecker, G. I. Japaridze, J. Klinovaja, and D. Loss, Phys. Rev. B 82, 045127 (2010).

[41] B. Karmakar, D. Venturelli, L. Chirolli, F. Taddei, V. Giovannetti, R. Fazio, S. Roddaro, G. Biasiol, L. Sorba, V. Pellegrini, and F. Beltram, Phys. Rev. Lett. 107, 236804 (2011).

[42] J. Klinovaja, P. Stano, and D. Loss, Phys. Rev. Lett. 109, 236801 (2012).

[43] G. L. Fatin, A. Matos-Abiague, B. Scharf, and I. Žutić, Phys. Rev. Lett. 117, 077002 (2016).

[44] A. Matos-Abiague, J. Shabani, A. D. Kent, G. L. Fatin, B. Scharf, and I. Žutić, Solid State Commun. 262, 1 (2017).

[45] L. N. Maurer, J. K. Gamble, L. Tracy, S. Eley, and T. M. Lu, Phys. Rev. Appl. 10, 054071 (2018).

[46] N. Mohanta, T. Zhou, J.-W. Xu, J. E. Han, A. D. Kent, J. Shabani, I. Žutić, and A. Matos-Abiague, Phys. Rev. Appl. 12, 034048 (2019).

[47] M. M. Desjardins, L. C. Contamin, M. R. Delbecq, M. C. Dartiailh, L. E. Bruhat, T. Cubaynes, J. J. Viennot, F. Mallet, S. Rohart, A. Thiaville, A. Cottet, and T. Kontos, Nat. Mater. 18, 1060 (2019).

[48] B. Braunecker, P. Simon, and D. Loss, Phys. Rev. B 80, 165119 (2009).

[49] C. P. Scheller, T.-M. Liu, G. Barak, A. Yacoby, L. N. Pfeiffer, K. W. West, and D. M. Zumbühl, Phys. Rev. Lett. 112, 066801 (2014).

[50] C.-H. Hsu, P. Stano, J. Klinovaja, and D. Loss, Phys. Rev. B 92 235435 (2015).

[51] See Supplemental Material at http://link.aps.org/supplemental/ 10.1103/PhysRevResearch.2.022052 for (i) details of derivation of bound state energy and wave function; (ii) an explicit form of the discretized Hamiltonian used in numerical calculations; (iii) details on stability of bound state to disorder; (iv) details on stability of localized channel in the Rashba layer to an in-plane magnetic field; (v) an alternative setup based on the rotating magnetic field; (vi) a mapping of interior branches to the Jackiw-Rebbi model.

[52] The obtained bound state is nontopological and different in origin from the well-known Jackiw-Rebbi mechanism that predicts a bound state with energy in the middle of the partial gap, arising from interior modes at $k=0$ and for smooth domain walls, 
see the SM [51]. However, such a bound state in the continuum [38] is not stable against disorder and easily hybridizes with the extended bulk modes coming from the ungapped exterior branches at $k= \pm 2 k_{\mathrm{so}}$.

[53] M. Serina, D. Loss, and J. Klinovaja, Phys. Rev. B 98, 035419 (2018).

[54] I. van Weperen, B. Tarasinski, D. Eeltink, V. S. Pribiag, S. R. Plissard, E. P. A. M. Bakkers, L. P. Kouwenhoven, and M. Wimmer, Phys. Rev. B 91, 201413(R) (2015).

[55] H. A. Nilsson, P. Caroff, C. Thelander, M. Larsson, J. B. Wagner, L.-E. Wernersson, L. Samuelson, and H. Q. Xu, Nano Lett. 9, 3151 (2009).

[56] J. Nitta, T. Akazaki, H. Takayanagi, and T. Enoki, Phys. Rev. Lett. 78, 1335 (1997).

[57] G. Engels, J. Lange, T. Schäpers, and H. Lüth, Phys. Rev. B 55, R1958(R) (1997).

[58] J. Schliemann, J. C. Egues, and D. Loss, Phys. Rev. Lett. 90, 146801 (2003).
[59] R. Winkler, Spin-orbit Coupling Effects in Two-Dimensional Electron and Hole Systems, Springer tracts in Modern Physics (Springer, Berlin, 2003).

[60] B. A. Bernevig, J. Orenstein, and S.-C. Zhang, Phys. Rev. Lett. 97, 236601 (2006).

[61] L. Meier, G. Salis, I. Shorubalko, E. Gini, S. Schön, and K Ensslin, Nat. Phys. 3, 650 (2007).

[62] M. Studer, G. Salis, K. Ensslin, D. C. Driscoll, and A. C. Gossard, Phys. Rev. Lett. 103, 027201 (2009).

[63] J. D. Koralek, C. P. Weber, J. Orenstein, B. A. Bernevig, S.-C. Zhang, S. Mack, and D. D. Awschalom, Nature (London) 458, 610 (2009).

[64] T. Meng, J. Klinovaja, and D. Loss, Phys. Rev. B 89, 205133 (2014).

[65] T. Cubaynes, M. R. Delbecq, M. C. Dartiailh, R. Assouly, M. M. Desjardins, L. C. Contamin, L. E. Bruhat, Z. Leghtas, F. Mallet, A. Cottet, and T. Kontos, npj Quant. Inform. 5, 47 (2019). 